\title{
ENSINO DE RELAÇÕES ENTRE FIGURAS E PALAVRAS IMPRESSAS COM EMPARELHAMENTO MULTIMODELO A CRIANÇAS COM AUTISMO
}

\section{TEACHING RELATIONS BETWEEN PICTURES AND PRINTED WORDS IN MATCHING WITH MULTIPLE SAMPLES TO CHILDREN WITH AUTISM}

\author{
Camila Graciela Santos Gomes \\ Faculdade de CIÊNCIAS MÉdicas de Minas GeRAIS, CEI DesenVolvimento Humano, BRASIL
}

\author{
ELENICE SEIXAS HANNA \\ UNIVERSIDADE dE BRASílIA E INSTITUTO NACIONAL DE CIÊNCIA E TECNOLOGIA SOBRE COMPORTAMENTO, COGNIÇÃO \\ E ENSINO, BRASIL
}

\author{
DEISY DAS GRAÇAS DE SOUZA \\ Universidade FEDERAL de SÃo CARLOS E INSTITUTO NACIONAL dE CIÊNCIA E TECNOLOGIA SOBRE \\ COMPORTAMENTO, COGNIÇÃO E ENSINO, BRASIL
}

\section{RESUMO}

Este estudo avaliou a eficácia de um procedimento adaptado de emparelhamento com o modelo (MTS multimodelo, com modelos compostos e seleção dos estímulos de comparação correspondentes a cada elemento do modelo) no ensino de relações arbitrárias e na emergência de relações derivadas. Foram ensinadas relações entre palavras impressas e figuras e nomeação de figuras, e avaliadas as relações derivadas nomeação oral das palavras impressas, emparelhamento entre palavras ditadas-palavras impressas e palavras impressas-figuras. Três participantes com autismo aprenderam as relações arbitrárias, nomearam as palavras impressas e apresentaram as relações derivadas para parte dos estímulos. Os resultados estendem os achados sobre a eficácia do procedimento multimodelo adaptado, utilizado anteriormente em emparelhamento de identidade, à aprendizagem de relações arbitrárias e à emergência de habilidades rudimentares de leitura nessa população.

Palavras-chave: aprendizagem relacional, emparelhamento com o modelo, modelos múltiplos, equivalência de estímulos, ensino de leitura, autismo

ABSTRACT
This study evaluated the efficacy of a multiple-sample matching-to-sample (MTS) procedure (MTS with compound samples and selection of all comparisons, each comparison corresponding to one element in the sample) for teaching arbitrary relations between pictures and printed words and to produce emergence of derived relations. Relations between printed words and pictures and picture naming were directly taught. Tests assessed derived oral naming of printed words, matching printed words to dictated words, matching printed words to pictures, and vice-versa. Three participants with autism learned the arbitrary relations, named printed words, and showed derived relations involving some of the stimuli. The results extend findings on the efficacy of the adapted multiple-sample procedure, previously used in identity matching, to learning of arbitrary relations and to the emergence of rudimentary reading skills in this population.

Key words: relational learning, matching-to-sample, multiple-sample stimuli, stimulus equivalence, reading acquisition, autism

\footnotetext{
O estudo é parte da dissertação de mestrado da primeira autora, que contou com bolsa da CAPES. Deisy G. de Souza é bolsista de Produtividade em Pesquisa do CNPq. As autoras são membros do Instituto Nacional de Ciência e Tecnologia sobre Comportamento, Cognição e Ensino, financiado pelo CNPq (Processo \#573972/2008-7) e pela FAPESP (Processo \# 2008/57705-8), que apoiou a redação do manuscrito. As autoras agradecem à Maria Isabel Pinheiro pela avaliação dos participantes e às famílias dos participantes do estudo. E-mail para correspondência: camila.gomes@ceidesenvolvimentohumano.com.br.
} 
Muitos conteúdos escolares abrangem a aprendizagem de relações entre estímulos, especialmente relações arbitrárias, que podem ser definidas como relações nas quais os estímulos envolvidos são fisicamente distintos (como ocorre tipicamente nas relações entre figuras e palavras impressas). Nesse caso, a relação entre os estímulos atende a critérios específicos estabelecidos arbitraria ou convencionalmente em cada comunidade verbal (Duarte \& de Rose, 2006). O ensino direto de algumas relações arbitrárias pode engendrar a emergência de outras relações arbitrárias que não foram diretamente ensinadas, ampliando assim a rede de relações entre os diversos estímulos. Esse processo de aprendizagem, que possibilita a geração de comportamentos novos pela derivação de comportamentos aprendidos e implica em grande economia no ensino, pode ser compreendido por meio do paradigma de equivalência de estímulos (Sidman, 1971, 1985, 1994; Sidman \& Tailby, 1982).

O ensino e a avaliação de relações condicionais arbitrárias entre estímulos podem ser conduzidos com diferentes tipos de tarefas. As tarefas de emparelhamento com o modelo (MTS) têm sido amplamente utilizadas (Carter \& Eckerman, 1975) porque são efetivas, claramente definidas e podem ser apresentadas em uma série de tentativas discretas, na quantidade que se mostrar necessária para gerar aprendizagem. No MTS típico, a tarefa é constituída por uma sequência de tentativas discretas, planejadas para ensinar relações entre dois conjuntos de estímulos; o conjunto dos estímulos modelo e o conjunto dos estímulos de comparação ou de escolha. Em cada tentativa são apresentados um estímulo modelo e dois ou mais estímulos de comparação (Cumming \& Berryman, 1961, 1965; de Rose, 2004). Para cada modelo, há apenas um estímulo de comparação correto, referido como estímulo discriminativo ou $\mathrm{S}+$; todos os outros estímulos de comparação apresentados simultaneamente são incorretos, referidos como estímulos negativos ou S-. Ao longo da série de tentativas, os diferentes estímulos modelo vão se alternado e o estímulo de comparação correto é aquele que se pretende que o aprendiz relacione ao estímulo modelo apresentado a cada tentativa. Em situação de ensino, as respostas de escolha são seguidas por consequências diferenciais, ou seja, repostas corretas são reforçadas e respostas incorretas não são seguidas de reforço (Saunders \& Green, 1999).

Dada a eficácia do MTS para ensinar relações entre estímulos e, sobretudo, para engendrar a emergência de relações novas, não diretamente ensinadas, derivadas de um conjunto de relações ensinadas (Sidman, 1994), seu emprego torna-se especialmente importante para o ensino de indivíduos com necessidades especiais, entre os quais indivíduos com Transtorno do Espectro do Autismo. Essa população é caracterizada por alterações na interação social, na comunicação, na linguagem e pela presença de comportamentos estereotipados (American Psychiatric Association, 2013). Além desses sintomas básicos, a literatura especializada relata que pessoas com autismo podem apresentar dificuldades em interpretar o que observam, em atribuir sentido além do literal e em associar palavras ao seu significado (Nation, Clarke, Wright, \&
Williams 2006). Essas dificuldades estão direta e/ou indiretamente relacionadas à aprendizagem de relações arbitrárias.

Apesar do uso amplamente disseminado do procedimento de MTS típico em situações de aplicação, com diversas populações (cf. Ghezzi, Williams, \& Carr, 1999; Martin \& Pear, 1978), a literatura especializada tem descrito dificuldades no ensino de relações condicionais arbitrárias a pessoas com autismo por meio desse recurso (Carr, Wilkinson, Blackman, \& McIlvane, 2000; Dube \& McIlvane, 1995; Eikeseth \& Smith, 1992; Kelly, Green, \& Sidman, 1998; O'Connor, Rafferty, Barnes-Holmes, \& Barnes-Holmes 2009; Vause, Martin, Yu, Marion, \& Sakko, 2005; Wilkinson, Rosenquist, \& McIlvane, 2009; Williams, Perez-González, \& Queiroz, 2005).

Alternativas ao MTS típico têm sido investigadas com populações com necessidades especiais (Serna, Dube, \& McIlvane, 1997; Stromer, Mcllvane, Dube, \& Mackay, 1993; Stromer, McIlvane, \& Serna, 1993), entre elas o uso do emparelhamento multimodelo ou MTS adaptado (Gomes \& de Souza, 2008; TEACCH, 1992) com pessoas com autismo. O MTS adaptado multimodelo apresenta, a cada tentativa, um estímulo modelo composto de tantos elementos quanto o número de estímulos de comparação e a tarefa do aprendiz é relacionar cada comparação ao seu respectivo elemento, componente do modelo composto. Essa tarefa corresponde à de ligar um item ao outro por meio de uma linha, como as usadas em situação escolar para relacionar, por exemplo, cada fruta de uma coluna de frutas, ao seu nome impresso em outra coluna (de nomes de frutas impressos). Um aspecto relevante para o ensino é que uma mesma tentativa pode ensinar múltiplas relações (tantas quantas forem os pares comparação-modelo).

Gomes e de Souza (2008) avaliaram o desempenho de 20 pessoas com autismo em tarefas de MTS por identidade, comparando a programação do MTS típico com o adaptado. No MTS típico, um estímulo modelo e três estímulos de comparação eram apresentados simultaneamente; a tarefa do participante era destacar um sinalizador e colocá-lo abaixo do comparação correto. No MTS adaptado, apresentavam-se três estímulos modelo (multimodelos) e três estímulos de comparação simultaneamente; a tarefa do participante era destacar cada um dos três comparações e colocá-los sobre os respectivos elementos do modelo. O objetivo do estudo consistia em avaliar se e como os participantes realizavam a tarefa (medir as relações de identidade, sem ensiná-las). A média de acertos foi significativamente maior nas tentativas de MTS adaptado do que no MTS típico, especialmente para os participantes que apresentavam dificuldade na tarefa.

Uma alternativa para favorecer a aprendizagem de relações arbitrárias é o uso combinado de estímulos modelo compostos e estímulos de comparação unitários (Dube, 1996; Stromer et al., 1993). O uso desse procedimento para ensinar a relação arbitrária entre estímulos pode tirar vantagem da discriminação condicional por identidade, ou seja, quando o estímulo de comparação correto é fisicamente igual a um dos componentes do modelo composto (supostamente, relações por identidade são facilmente aprendidas devido à 
similaridade física entre os estímulos a serem relacionados). Por exemplo, o modelo pode apresentar uma figura e a palavra impressa correspondente; a matriz de comparações ora apresenta apenas figuras (entre as quais a que é igual à apresentada como um dos componentes do modelo), ora apenas a palavra impressa (que é igual ao outro componente do modelo). Para realizar a tarefa, o aprendiz tem que atentar para todos os componentes do estímulo modelo, uma vez que apenas um deles estará presente na matriz de comparações. Esta exigência parece favorecer o comportamento de rastrear tanto o estímulo modelo como os estímulos de comparação. Depois que a tarefa está bem estabelecida, geralmente verifica-se que o aprendiz é capaz não só de relacionar os elementos iguais, mas também os diferentes. Por exemplo, um teste consiste em apresentar o modelo apenas com a figura ou apenas com a palavra impressa; além de responder por identidade (quando modelos e comparações são figuras ou quando ambos são palavras impressas), o aprendiz geralmente consegue relaciona-los quando os estímulos de comparações são fisicamente diferentes do modelo (a figura modelo com a palavra impressa correspondente ou a palavra impressa modelo com a figura correspondente).

O objetivo do presente estudo foi ampliar a avaliação de tarefas de MTS adaptado multimodelo para o ensino de relações arbitrárias entre figuras e palavras impressas. Conduzido com crianças com autismo, o estudo avaliou a eficácia do procedimento de emparelhamento multimodelo (um modelo para cada comparação) com modelos compostos (Dube, 1996; Stromer et al., 1993, 1997), tanto para ensinar as relações arbitrárias quanto para gerar a emergência de comportamentos novos.

Foram ensinadas duas tarefas: o emparelhamento com o modelo entre figuras e palavras impressas (relações puramente visuais), por meio do uso de estímulos compostos, e a nomeação de figuras. Além de avaliar a aprendizagem do que foi diretamente ensinado, o estudo também avaliou a emergência de desempenhos novos: a nomeação oral das palavras impressas, a seleção de palavras impressas quando os estímulos modelo eram palavras ditadas e a relação entre palavras impressas e figuras (e vice-versa), quando os modelos deixavam de ser compostos. A racional para os testes era que se as figuras se tornassem equivalentes às palavras impressas (por meio do emparelhamento) e se os participantes nomeassem as figuras, então, por derivação, eles poderiam dizer a mesma palavra para a figura e para a palavra impressa correspondente e identificar a palavra impressa quando o modelo fosse uma palavra falada.

A aprendizagem de relações arbitrárias e a emergência de relações novas podem ocorrer com quaisquer tipos de estímulos, mas quando os estímulos são palavras faladas, palavras impressas e as figuras correspondentes, como os escolhidos para este estudo, os desempenhos emergentes caracterizam o que se pode chamar de leitura receptiva, leitura com compreensão e comportamento textual (de Rose, de Souza, \& Hanna, 1996; Sidman, 1971; Skinner, 1957), o que implica uma vantagem adicional para a população de indivíduos com autismo.

\section{Participantes \\ MÉTODO}

Participaram do estudo três meninos com diagnóstico prévio de autismo (Tabela 1), falantes, não alfabetizados, com idades entre cinco anos e cinco meses e 12 anos e dois meses. Dois participantes apresentavam autismo grave e um apresentava autismo leve/moderado, com referência na Childhood Autism Rating Scale - CARS (Schopler, Reichler, \& Renner, 1988; estudo de validação por Pereira, Riesgo, \& Wagner, 2008) e todos apresentavam atraso no desenvolvimento e alterações nos comportamentos, de acordo com o Psychoeducational Profile-Revised PEP-R (Schopler, Reichler, Bashford, Lansing, \& Marcus, 1990; para discussão ver Leon, Bosa, Hugo, \& Hutz, 2005). O estudo foi aprovado pelo Comitê de Ética em Pesquisa em Seres Humanos da Universidade Federal de São Carlos (Parecer número 234/2006).

\section{Situação e Materiais}

As sessões, com duração média de 50 minutos, eram realizadas em uma sala com poucos estímulos, mobiliada com uma mesa, duas cadeiras e uma filmadora. As tarefas eram conduzidas na mesa, utilizando-se figuras em tamanho $10 \mathrm{~cm}$ x $10 \mathrm{~cm}$ com velcro na parte posterior e pares de palavras impressas em letras maiúsculas, fonte Arial, tamanho 72 e velcro colado na parte posterior de uma palavra de cada par. Uma base de papel de $10 \mathrm{~cm}$ x $15 \mathrm{~cm}$ (LxA), com velcro na parte anterior, servia para delimitar o campo da tarefa e as áreas onde os estímulos eram posicionados. Folhas de papel com velcro foram montadas em um fichário para organizar as tentativas que requeriam a seleção e a nomeação de palavras impressas. Um marcador, de $5 \mathrm{~cm} \mathrm{x} 1 \mathrm{~cm}$, foi confeccionado em papel com velcro na parte posterior, e era usado para indicar a escolha nas tentativas que requeriam a seleção de palavras; o velcro do marcador podia ser preso ao velcro da palavra.

As palavras utilizadas para cada participante foram escolhidas a partir de uma lista produzida pelos responsáveis pela criança (em função do uso ou do interesse) e eram constituídas, na maioria dos casos, por sílabas simples (consoante-vogal) e facilmente representadas em imagem. Os reforçadores utilizados nas sessões experimentais (brinquedos e consequências sociais preferidas pela criança) foram escolhidos a partir de sugestões dos responsáveis.

O posicionamento do participante e do experimentador na sala era estrategicamente planejado para restringir o acesso do participante aos estímulos da pesquisa. À esquerda do participante havia uma parede, à sua frente havia uma mesa e o experimentador estava localizado atrás do participante. 
Tabela 1

Idade, Pontuação na CARS, Pontuação do Desenvolvimento no PEP-R, e Perfil na Escala de Comportamento do PEP-R de Cada Participante

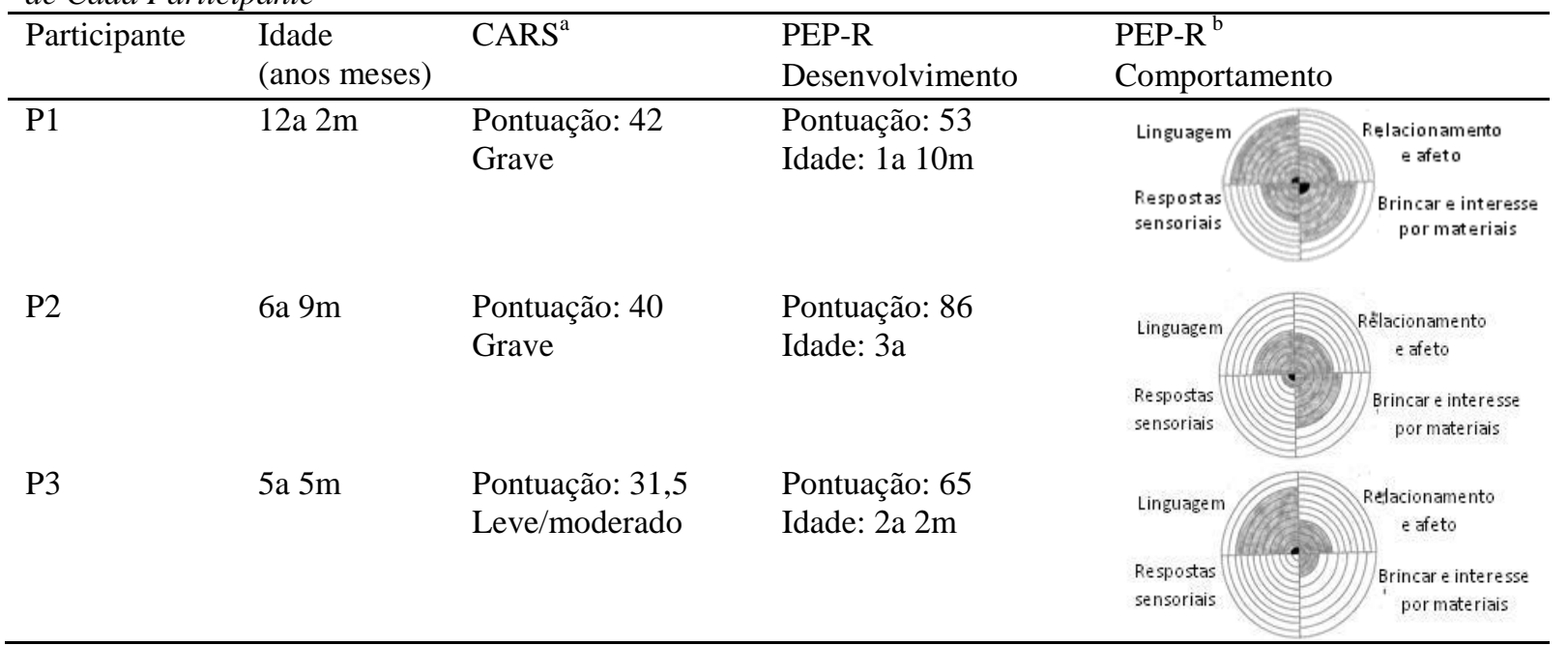

${ }^{\text {a }}$ Escala CARS: Normal: 15 a 29,5; Leve/Moderado: 30 a 36,5; Grave: 37 a 60.

${ }^{\mathrm{b}}$ Escala PEP-R de comportamento: marcações em preto indicam alterações graves; marcações em cinza alterações moderadas; espaços em branco indicam ausência de comprometimento.

\section{Procedimentos}

Foram ensinadas com MTS adaptado (multimodelo), as relações condicionais entre estímulos modelo compostos (figura e palavra impressa) e estímulos de comparação unitários (palavras impressas), chamadas neste estudo de relações BC-C (B designa o conjunto de figuras e $\mathrm{C}$ designa o conjunto de palavras impressas; diferentes letras podem ser, e tem sido usadas, em diferentes estudos). A nomeação de figuras (B-D, onde D designa palavras faladas pelo participante) também foi ensinada. Testes conduzidos em extinção verificavam as relações entre figuras e palavras impressas e vice-versa
(B-C e C-B), a nomeação de palavras impressas (C-D) e as relações entre palavras ditadas e palavras impressas (A-C, onde $\mathrm{A}$ indica palavra ditada pelo experimentador), nesta ordem.

A Figura 1 apresenta o diagrama das relações ensinadas e testadas, com a ilustração de uma tentativa de cada tipo. As relações ensinadas são representadas por setas cheias e as relações testadas são representadas pelas setas tracejadas. A seta cheia mais larga indica que a relação A-B (emparelhamento entre palavras ditadas e figuras) já estava presente no repertório dos participantes.

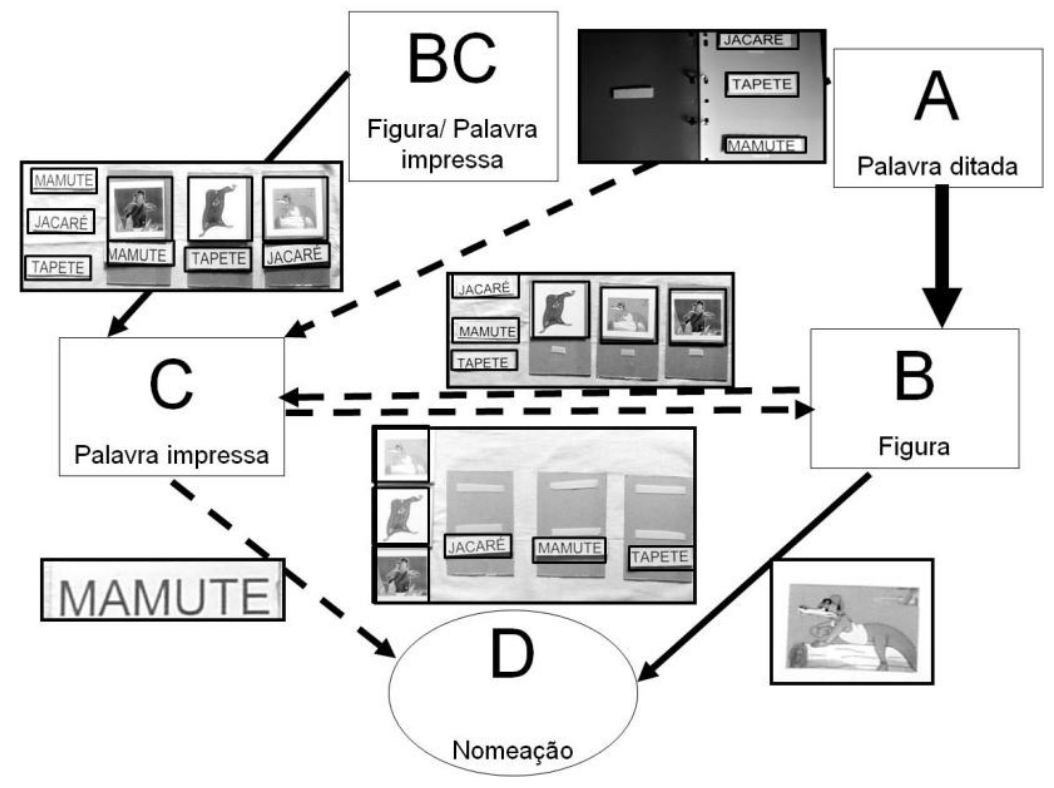

Figura 1. Rede de relações condicionais envolvidas no procedimento utilizado. As setas partem do conjunto dos estímulos modelo para o conjunto dos estímulos de comparação. Setas com linhas cheias representam desempenhos ensinados (BC-C e B-D). Setas com linhas tracejadas representam desempenhos testados (B-C, C-B, A-C e C-D). A seta cheia mais grossa representa desempenho que as crianças apresentavam antes do início do procedimento (A-B). 
A relação A-B foi verificada em um teste préexperimental com MTS típico, no qual os participantes apresentaram $100 \%$ de acertos (considerando-se que foi previamente identificado o interesse dos participantes por estes itens). Nesse teste, programou-se uma tentativa para cada estímulo, com a apresentação do estímulo modelo (nome da figura ditado pela pesquisadora) e três estímulos de comparação (figuras) dispostos na mesa. As outras relações (B-C, C-B, C-D e A-C) foram pré-testadas e os participantes não apresentaram acertos.

Um delineamento de linha de base múltipla foi utilizado em duas unidades, cada uma com três conjuntos de dois a três estímulos diferentes. Em cada unidade realizavam-se atividades de ensino com o Conjunto 1 de estímulos e avaliações com esses e outros estímulos não ensinados (dos Conjuntos 2 e 3); em seguida, o mesmo era feito com o Conjunto 2 e, então, com o Conjunto 3 , sempre avaliando, antes e depois do ensino de cada conjunto, todas as palavras da unidade.
A Tabela 2 apresenta os conjuntos de palavras utilizados em cada unidade para cada participante. A maioria das palavras selecionadas era constituída por sílabas simples (consoante-vogal), com duas ou mais letras diferentes, geralmente as consoantes. As palavras impressas de cada um dos conjuntos eram do mesmo tamanho para evitar que os participantes ficassem sob controle da extensão das palavras e não da relação figurapalavra; as palavras impressas diferiam de tamanho de um conjunto para outro. O uso de palavras diferentes para cada criança levou em conta seu repertório de entrada e fatores de natureza motivacional.

Tentativas de revisão, com a função de favorecer a manutenção das relações aprendidas com conjuntos de estímulos anteriores, eram realizadas no final das atividades de cada conjunto, a partir do Conjunto 2. Eram programadas três tentativas de revisão BC-C e uma tentativa B-D com cada figura.

Tabela 2

Conjuntos de Palavras e Consequências Utilizadas em cada Unidade, para cada Participante

\begin{tabular}{|c|c|c|c|c|}
\hline Participantes & Unidade & Conjuntos & Palavras & Consequências \\
\hline \multirow[t]{2}{*}{$\mathrm{P} 1$} & 1 & $\begin{array}{l}1 \\
2 \\
3\end{array}$ & $\begin{array}{l}\text { rede, pato, bola } \\
\text { Rafiza, Camila, pirata } \\
\text { galo, meia, fada }\end{array}$ & Elogios, palmas e brincadeiras \\
\hline & 2 & $\begin{array}{l}1 \\
2 \\
3\end{array}$ & $\begin{array}{l}\text { tapete, mamute, jacaré } \\
\text { Zezé, Bidu, foto } \\
\text { gênio, Tênis, clube }\end{array}$ & \\
\hline \multirow[t]{2}{*}{$\mathrm{P} 2$} & 1 & $\begin{array}{l}1 \\
2 \\
3\end{array}$ & $\begin{array}{l}\text { Raul, bola, suco } \\
\text { carro, fanta, Edgar } \\
\text { música, Bianca, picolé }\end{array}$ & $\begin{array}{c}\text { Tentativas organizadas como um jogo } \\
\text { e pontos }\end{array}$ \\
\hline & 2 & $\begin{array}{l}1 \\
2 \\
3\end{array}$ & $\begin{array}{l}\text { galo, água, xixi } \\
\text { batata, jacaré, tomate } \\
\text { vaca, pera, moto }\end{array}$ & \\
\hline \multirow[t]{2}{*}{ P3 } & 1 & $\begin{array}{l}1 \\
2 \\
3\end{array}$ & $\begin{array}{l}\text { Cheetos, pirulito } \\
\text { mamãe, papai } \\
\text { bola, vaca }\end{array}$ & Elogios, palmas e brincadeiras \\
\hline & 2 & $\begin{array}{l}1 \\
2 \\
3\end{array}$ & $\begin{array}{l}\text { Victor, jacaré, batata } \\
\text { moto, água, vovó } \\
\text { xixi, pato, suco }\end{array}$ & \\
\hline
\end{tabular}

Ensino das relações BC-C e B-D.

Cada tentativa de ensino das relações BC-C com MTS adaptado era iniciada com a apresentação de três modelos compostos BC (figura e palavra impressa) dispostos horizontalmente no centro da mesa e os três comparações posicionados à esquerda dos modelos (Figura 1, painel superior à esquerda). O participante era orientado a parear cada comparação com seu respectivo modelo, ou seja, escolher, à esquerda, o comparação igual a um dos elementos do modelo composto, retirá-lo e posicioná-lo sobre o modelo. Eram realizadas três tentativas BC-C com os estímulos de um conjunto e, então, iniciavam-se as tentativas B-D com a apresentação de uma figura sobre a mesa e a instrução "Que figura é esta?"; o participante deveria nomear oralmente cada figura. A organização e a sequência de apresentação dos estímulos eram semi-aleatórias nos dois tipos de tentativa.

Respostas corretas em tentativas B-D e BC-C eram reforçadas imediatamente por consequências sociais (palmas, elogios ou pontos; ver Tabela 2) para todos os participantes. Nas tentativas BC-C a consequência era contingente a cada pareamento (isto é, a cada resposta de seleção, até que todos os elementos fossem emparelhados) e nas tentativas B-D era contingente a cada resposta de nomeação.

Nas tentativas BC-C, quando o participante errava, a pesquisadora auxiliava fisicamente na correção 
(auxiliava o participante a trocar os estímulos de lugar e solicitava verbalmente que ele prestasse atenção); nas tentativas B-D, quando o participante nomeava uma figura de maneira inadequada ou não a nomeava, a pesquisadora apresentava o modelo oral e instruía o participante a repeti-lo (treino de comportamento ecoico, na presença da figura). A ajuda era retirada quando o participante apresentava acertos.

Após duas tentativas B-D e três tentativas do tipo BC-C com cada uma das figuras de um conjunto (total de nove tentativas), a criança tinha a oportunidade de participar de brincadeiras, que eram específicas para ela, em função de suas preferências. $\mathrm{O}$ ensino de cada três pares de figura-palavra (ver Tabela 2) era repetido pelo menos três vezes (nove tentativas), assim como o ensino de B-D (seis tentativas repetidas por três vezes, totalizando 18 tentativas), antes da realização dos testes. A etapa de ensino terminava quando o participante: (a) realizava, pelo menos, nove tentativas BC-C (sendo que as três últimas deveriam estar corretas) e 18 tentativas B-D (sendo que as seis últimas deveriam estar corretas); e (b) $100 \%$ de acertos no Teste B-C, que era requisito para a realização dos outros testes.

\section{Procedimentos alternativos de correção: ensino integrado das relações B-D/BC-C. \\ Quando os participantes não aprendiam a relação} B-C com o procedimento descrito, em três sessões de ensino, procedimentos alternativos (de correção) eram utilizados. Nesses procedimentos, realizava-se o ensino integrado das relações B-D/BC-C (Figura 2) em três tentativas consecutivas. $\mathrm{O}$ ensino de B-D antes de BC-C, além de ensinar a nomeação da figura, aumentava as chances do participante observar a figura apresentada em seguida no modelo composto (BC) e de seu controle sobre a resposta de escolha da palavra impressa, mesmo que inicialmente fosse uma escolha por identidade.

Inicialmente eram apresentadas as figuras (B) com os estímulos de comparação (C) virados para baixo, de forma que o participante não podia ver as palavras impressas. As palavras dos modelos compostos estavam cobertas por uma tira de papel (Figura 2, Painel 1). Diante das figuras, o participante era instruído a nomeá-las oralmente (ensino de B-D e resposta de observação). Em seguida, a tira de papel era retirada e o participante era instruído a olhar para cada figura e sua respectiva palavra impressa (Figura 2, Painel 2). Depois disso, a tira de papel voltava a cobrir as palavras componentes dos modelos, os estímulos de comparação (palavras impressas) eram mostrados do lado esquerdo (Figura 2, Painel 3) e o participante deveria escolher a palavra correspondente a cada figura do modelo (Figura 2, Painel 4). Após o participante ter colocado os estímulos de comparação junto aos estímulos modelo, a tira de papel era retirada e as relações entre os estímulos eram conferidas com o participante (Figura 2, Painel 5). Procedimentos de ajuda eram utilizados, quando necessário, para direcionar a atenção dos participantes para os estímulos a serem observados. Para isso, a pesquisadora pegava a mão dominante do participante, apontava com ela para os estímulos a serem observados, enquanto segurava a outra mão sobre a mesa para evitar que ele se distraísse com estímulos irrelevantes. Além disso, a pesquisadora instruía o participante a observar os estímulos (exemplo: "olha para a figura; agora olha para a palavra"), perguntava se ele tinha olhado para as figuras e para as palavras e se ela poderia cobrir as palavras. As consequências para acertos e erros eram iguais às utilizadas no ensino intercalado das relações B-D e BC-C.

No procedimento de correção, o ensino integrado das relações B-D/BC-C era realizado desde o início com três estímulos modelo e três comparações. Se, ainda assim, um participante não aprendesse a relação $\mathrm{B}-\mathrm{C}$, o número de itens nas tentativas era reduzido para dois modelos e dois comparações na Unidade 1 e, em seguida, ampliado para três modelos e três comparações na Unidade 2.

Os critérios para finalização da etapa de ensino com procedimento de correção eram: (1) a realização de pelo menos seis tentativas de ensino integrado B-D/BC-C (sendo que as três últimas deveriam estar corretas); e (b) $100 \%$ de acertos no Teste B-C.

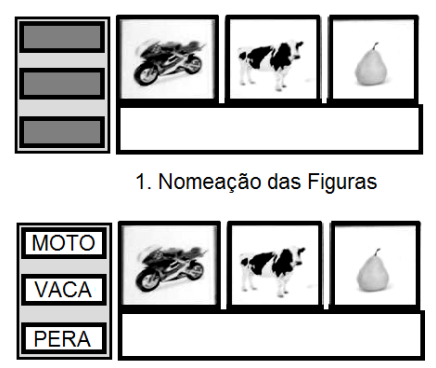

3. Apresentação dos comparações

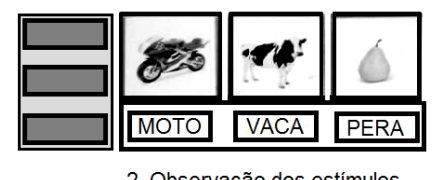

2. Observação dos estímulos

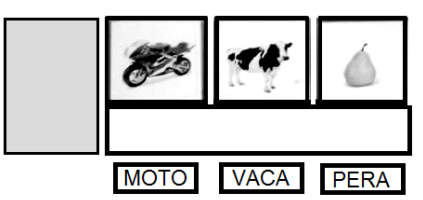

4. Realização da tentativa

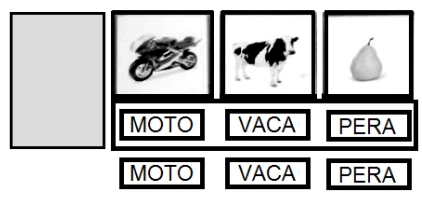

5. Conferência

Figura 2. Exemplo de tentativa de ensino B-D/ BC-C: 1) apresentação apenas das figuras para nomeação (B-D); 2) apresentação das palavras embaixo das respectivas figuras - instrução ao participante para olhar cada par figura-palavra impressa; 3) apresentação dos estímulos de comparação à esquerda das figuras para o pareamento na ausência do elemento impresso do modelo; 4) participante realiza a escolha das palavras e coloca abaixo das figuras; 5) as palavras corretas são apresentadas para conferência junto com a resposta do participante.

\section{Sondas de relações emergentes.}

Para cada conjunto de três estímulos, realizava-se um conjunto de testes, na seguinte ordem: emparelhamento de palavras impressas a figuras (B-C), emparelhamento de figuras a palavras impressa (C-B), nomeação de palavras impressas (C-D) e emparelhamento palavras impressas as palavras ditadas (A-C). Em todos os testes, a organização e a sequência de apresentação dos estímulos eram semi-aleatórias e não havia consequências diferencias para erros ou acertos.

O Teste B-C era conduzido em três tentativas de MTS adaptado, em que cada elemento do modelo era uma figura (sem a correspondente palavra impressa, que era 
apresentada somente como um dos comparacões), nas quais o participante deveria apresentar $100 \%$ de acertos para que os outros testes pudessem ser realizados (caso o participante apresentasse erros neste teste, o procedimento retornava às tentativas de ensino).

Em seguida, eram realizadas três tentativas de Teste C-B, também com MTS adaptado, no qual cada elemento do modelo composto era uma palavra impressa (sem a figura correspondente, que só era apresentada entre os comparações).

Seguiam-se duas tentativas de nomeação C-D (leitura) para cada palavra: a palavra era apresentada sozinha sobre a mesa e a experimentadora perguntava: "Que palavra é esta? ou "O que está escrito?".

$\mathrm{O}$ conjunto de testes era encerrado com um bloco de tentativas A-C com MTS típico em que o modelo era uma palavra ditada e os três estímulos de comparação, apresentados simultaneamente, eram palavras impressas, apresentadas presas com velcro em folhas de papel, montadas em um fichário. A pesquisadora ditava a palavra e o participante deveria pegar o marcador na página à esquerda do fichário e colocar à direita, abaixo da palavra impressa escolhida (ver Figura 1). Eram realizadas duas tentativas com cada modelo ditado.

Além dos testes relativos a cada conjunto de estímulos, também eram realizados, respectivamente, no início e no final de cada conjunto, o Pré e o Pós-teste de nomeação de palavras (C-D) da unidade, nos quais as tentativas eram apresentadas por meio de um fichário e as palavras encontravam-se presas com velcro, uma em cada página. Cada palavra da unidade era testada em uma tentativa.

Todas as sessões eram filmadas e o número de tentativas de ensino, de acertos e de erros eram registrados pela pesquisadora durante as sessões e conferidos posteriormente a cada sessão, a partir das filmagens. Dois observadores independentes e ingênuos quanto ao propósito da pesquisa registraram $30 \%$ das sessões. O coeficiente de fidedignidade, calculado como número de acordos dividido pelo total de acordos e desacordos e multiplicado por 100 (Kazdin, 1982), foi de 100\%. Foram registradas as respostas dos participantes nas tentativas de ensino e nas tentativas de testes de relações emergentes.

Tabela 3

Número de Tentativas Realizadas para Alcançar o Critério de Aprendizagem de cada Relação com os Conjuntos de Estímulos de Cada Unidade, Para Cada Participante

\begin{tabular}{|c|c|c|c|c|c|c|c|c|c|c|}
\hline \multirow{3}{*}{$\begin{array}{l}\text { Uni- } \\
\text { dade }\end{array}$} & \multirow{3}{*}{$\begin{array}{l}\text { Con- } \\
\text { junto }\end{array}$} & \multicolumn{2}{|c|}{ P1 } & \multicolumn{3}{|c|}{$\mathrm{P} 2$} & \multicolumn{4}{|c|}{ P3 } \\
\hline & & BC-C & B-D & BC-C & B-D & B-D/ BC-C & BC-C & B-D & B-D/BC-C & B-D/ BC-C \\
\hline & & & & & & 3 modelos & & & 3 modelos & 2 e 3 modelos \\
\hline \multirow[t]{3}{*}{1} & 1 & 9 & 18 & 117 & 102 & 18 & 63 & 78 & 60 & 36 \\
\hline & 2 & 9 & 24 & - & - & 9 & - & - & - & 48 \\
\hline & 3 & 9 & 18 & - & - & 9 & - & - & - & 6 \\
\hline \multirow[t]{3}{*}{2} & 1 & 9 & 18 & - & - & 9 & - & - & - & 6 \\
\hline & 2 & 9 & 18 & - & - & 15 & - & - & - & 18 \\
\hline & 3 & 9 & 18 & - & - & 6 & - & - & - & 15 \\
\hline
\end{tabular}

Nota: BD/BC-C indica o procedimento alternativo de correção que combinava os dois tipos de relações, iniciando pelo ensino de nomeação de figuras.

\section{RESULTADOS}

A Tabela 3 apresenta o número de tentativas realizadas para cada conjunto de estímulos, das duas unidades de ensino, para cada participante. O participante P1 aprendeu as relações diretamente ensinadas, no geral, com nove tentativas BC-C e 18 tentativas B-D em cada conjunto de estímulos (exceto no Conjunto 2 da Unidade 1). O participante $\mathrm{P} 2$ realizou 117 tentativas $\mathrm{BC}-\mathrm{C}$ e 102 de B-D, no Conjunto 1 da Unidade 1, em três sessões, e não aprendeu a relação B-C. Por essa razão, foi necessário o uso do procedimento alternativo de ensino integrado BD/BC-C. Nesse caso ele precisou, em média, de 11 tentativas para aprender a relação B-C em cada conjunto das duas unidades. $\mathrm{O}$ participante $\mathrm{P} 3$ realizou 63 tentativas BC-C e 78 de B-D, no Conjunto 1 da Unidade 1, em três sessões, e não aprendeu a relação B-C; foi necessário o uso do procedimento alternativo de ensino integrado $\mathrm{B}$ D/BC-C, porém o participante não aprendeu a relação B-C após 60 tentativas realizadas em três sessões. Foi necessária a diminuição do número de estímulos nas tentativas B-D/BC-C, nos conjuntos da Unidade 1, porém, na Unidade 2 isso não foi necessário; o participante precisou, em média, de 30 tentativas em cada bloco da primeira unidade e de 13 em cada bloco da segunda unidade. De maneira geral, os participantes apresentaram poucos erros e não demonstraram dificuldades na realização das tentativas de nomeação ou de MTS multimodelo. As tentativas de ensino foram repetidas, na maioria das vezes, porque os participantes não apresentaram a relação B-C nos testes iniciais, mesmo tendo realizado as tentativas de ensino corretamente.

A Figura 3 mostra as porcentagens de acertos nos testes com os conjuntos de estímulos de cada unidade. As porcentagens de acertos no Teste B-C são representadas por barras cinza; as porcentagens de acertos no Teste C-B são representadas por barras cinza escuro; as porcentagens de acertos no Teste C-D são representadas por barras com textura pontilhada; Finalmente, as porcentagens de acertos no Teste A-C são representadas por barras pretas. Os resultados dos testes com os diferentes conjuntos de 
estímulos também apresentam diferenças entre os participantes.

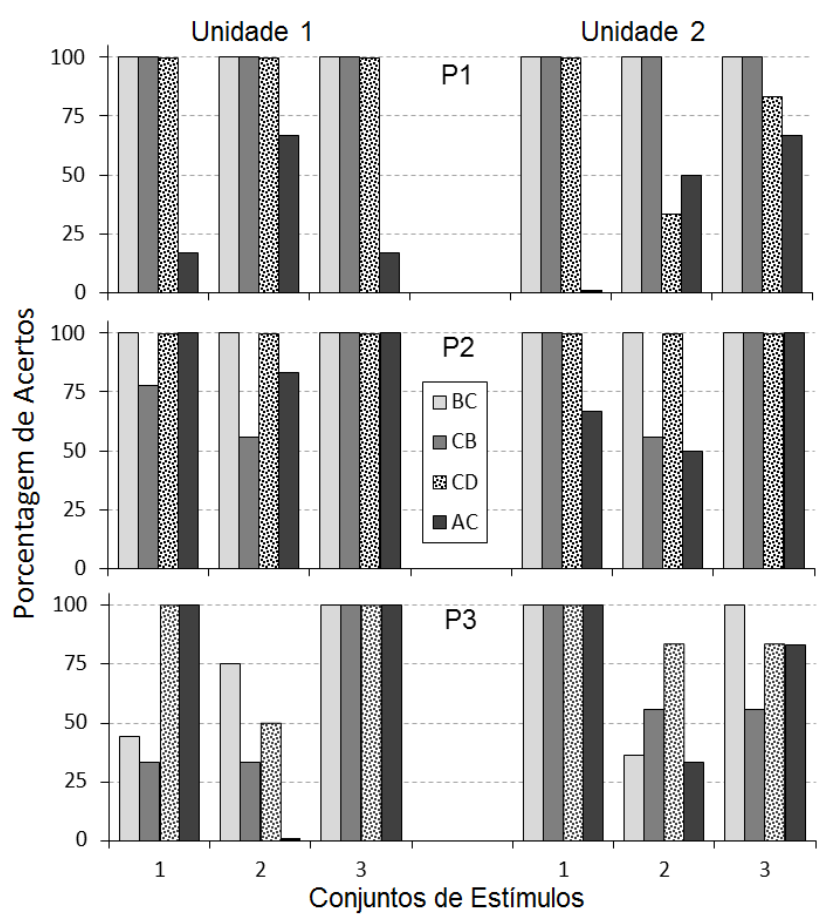

Figura 3. Porcentagem de acertos em Testes B-C, C-B, C$\mathrm{D}$ e A-C de cada conjunto de estímulos das Unidades 1 (à esquerda) e 2 (à direita), para cada participante.

Após apresentar $100 \%$ de acertos nas tentativas de treino BC-C, o participante P1 apresentou $100 \%$ de acertos em todas as tentativas de pareamento entre figuras e palavras (B-C e C-B) e de nomeação das palavras impressas em quatro dos seis conjuntos de estímulos. $\mathrm{O}$ desempenho nas tentativas de pareamento entre palavra ditada e escrita (A-C) foi, entretanto, sistematicamente baixo ao longo dos conjuntos de estímulos das duas unidades. Para quatro conjuntos, as porcentagens foram menores que $50 \%$ de acertos.

$\mathrm{O}$ participante $\mathrm{P} 2$ apresentou desempenhos perfeitos (100\% de acertos) nos Testes B-C e C-D ao longo das duas unidades e em três dos seis conjuntos de estímulos dos Testes C-B e A-C. Erros ocorreram com três conjuntos de estímulos nos Testes C-B e A-C, mas os acertos foram maiores que $50 \%$.
Os desempenhos de P3 nos testes foram, em geral, inferiores aos dos outros dois participantes e apresentaram maior variabilidade. Este participante apresentou desempenho acurado em metade dos conjuntos de estímulos, nos Testes B-C, C-D e A-C. No Teste A-C, o participante obteve $100 \%$ de acertos em dois dos seis conjuntos (Conjunto 3 da Unidade 1 e Conjunto 1 da Unidade 2). Os escores nos conjuntos em que P3 apresentou erros variaram de $36,1 \%$ a $75,0 \%$ no Teste BC, de $33,3 \%$ a $55,6 \%$ no Teste C-B, de $50,0 \%$ a $83,3 \%$ no Teste C-D e de $0 \%$ a $83,3 \%$ de acerto no Teste A-C.

A Figura 4 mostra o desempenho dos participantes P1, P2 e P3 em Testes de nomeação (C-D) de cada palavra impressa da Unidade 1 (gráficos à esquerda) e da Unidade 2 (gráficos à direita). A linha tracejada indica, de acordo com o delineamento de linha de base múltipla entre conjuntos de palavras, quando as atividades de ensino foram realizadas com cada conjunto. Tanto na Unidade 1 quanto na Unidade 2, os participantes não nomeavam as palavras impressas antes que elas fossem ensinadas (Avaliação 1, pré-teste do Conjunto 1), porém, após o ensino do primeiro conjunto, os participantes passaram a nomear as palavras trabalhadas, mas não nomearam as outras palavras que ainda não tinham sido ensinadas (Avaliação 2, pós-teste do Conjunto 1). $\mathrm{Na}$ avaliação que precedeu o ensino do segundo conjunto (Avaliação 3, pré-teste do Conjunto 2), os participantes mostraram retenção, nomeando corretamente as palavras trabalhadas no conjunto anterior, porém não nomearam as outras palavras não ensinadas. Na avaliação após o ensino do Conjunto 2 (Avaliação 4, pós-teste do Conjunto 2), os participantes, em geral, mantiveram a nomeação correta das palavras do Conjunto 1 e passaram a nomear as palavras do segundo conjunto no pós-teste deste conjunto (exceto P2, que não nomeou as palavras na Avaliação 4a com o uso do fichário, mas nomeou no Teste $4 \mathrm{~b}$, sem o fichário). $\mathrm{Na}$ avaliação antes do ensino das palavras do Conjunto 3 (Avaliação 5, pré-teste do Conjunto 3), os participantes nomearam corretamente as palavras impressas ensinadas nos conjuntos anteriores (retenção), mas não nomearam os itens que seriam introduzidos neste conjunto; na avaliação após o ensino no Conjunto 3 (pósteste do Conjunto 3), os participantes P1 e P2 nomearam todas as palavras trabalhadas nos três conjuntos e P3 nomeou a maioria delas. Em resumo, os resultados apresentados na Figura 4 mostram que os participantes aprenderam a nomear as palavras impressas após serem expostos às atividades de ensino. 

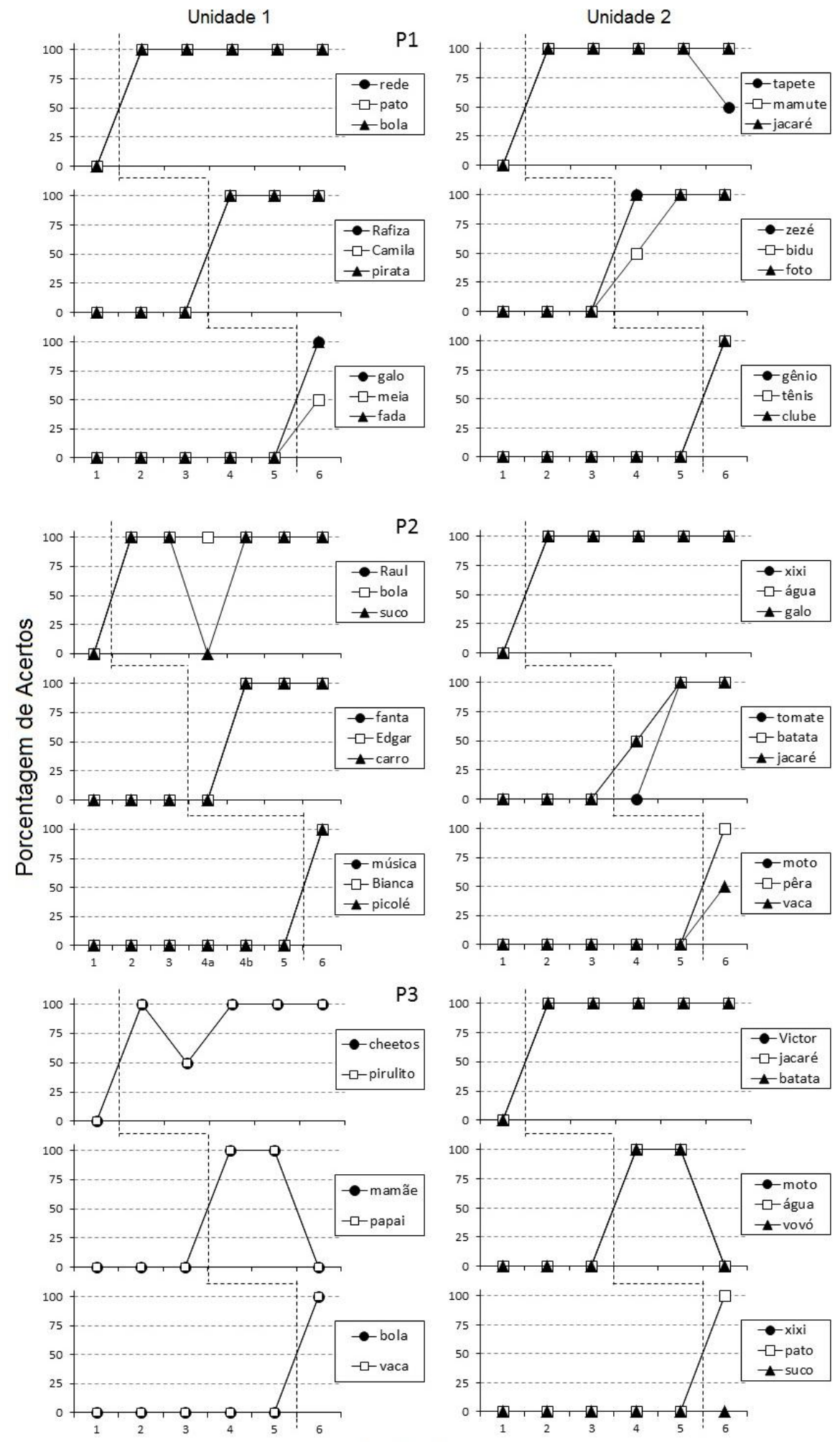

Avaliações

Figura 4. Porcentagem de acertos nas sucessivas avaliações de Linha de Base de Nomeação Oral das palavras impressas em Testes C-D da Unidade 1 (Esquerda) e da Unidade 2 (Direita). As Avaliações 1, 3 e 5 referem-se à nomeação de palavras impressas dos Conjuntos 1, 2 e 3 na fase de Linha de Base (Pré-Testes). As Avaliações 2, 4 e 6 referem-se à nomeação das mesmas palavras após o ensino de cada conjunto de palavras (Pós-Testes). As linhas pontilhadas indicam a introdução do ensino de cada conjunto de três palavras, de acordo com o delineamento de linha de base múltipla entre conjuntos. 


\section{DISCUSSÃO}

O objetivo geral do estudo foi verificar se o ensino de relações arbitrárias, por meio do emparelhamento multimodelo ou MTS adaptado, e o ensino de nomeação de figuras poderiam favorecer a aprendizagem de relações diretamente ensinadas e a emergência de relações não ensinadas por participantes com autismo, no contexto do ensino de habilidades rudimentares de leitura. De maneira específica, o estudo pretendia ensinar os participantes a nomear oralmente algumas palavras impressas (leitura oral ou comportamento textual); a identificar as palavras impressas diante das correspondentes palavras ditadas (leitura receptiva); e a relacionar as palavras impressas a figuras, assim como o inverso (habilidades rudimentares de compreensão de palavras impressas).

As condições especialmente planejadas para esse estudo incluíram: (1) o emprego do emparelhamento multimodelo e o ensino de relações visuais-visuais; (2) a utilização de modelos compostos e comparações unitários, de modo que o ensino podia ter um componente de emparelhamento por identidade entre um elemento do modelo e o estímulo de comparação correto, mas também podia engendrar o emparelhamento arbitrário entre os dois componentes do modelo por ocasião da resposta de escolha de um deles sob controle da identidade; (3) o ensino da nomeação de estímulos; (4) o uso de estímulos familiares.

Todos os participantes aprenderam as relações arbitrárias entre figuras e palavras impressas (B-C), ensinadas diretamente por meio do emparelhamento multimodelo. O participante $\mathrm{P} 1$ aprendeu as relações BC$\mathrm{C}$ e B-D sem necessidade de procedimentos remediativos e com o número mínimo de tentativas de ensino programado. Os participantes $\mathrm{P} 2$ e $\mathrm{P} 3$ necessitaram de alterações no procedimento de ensino e, após tais mudanças, o número de tentativas de ensino realizadas por P2 ficou próximo ao número mínimo programado. O participante P3, por sua vez, necessitou de um número de tentativas, que foi duas vezes e meia maior do que o número mínimo programado. A quantidade de tentativas de ensino realizadas pelos participantes desse estudo é relativamente baixa se comparada a outros estudos que pretenderam ensinar relações arbitrárias entre estímulos visuais a participantes com autismo (Carr et al., 2000; Dube \& McIlvane,1995; Eikeseth \& Smith, 1992; Vause et al., 2005). Eikeseth e Smith (1992), por exemplo, precisaram de 360 e 1546 tentativas, na primeira parte do estudo, para ensinar duas relações visuais-visuais entre letras gregas e os nomes impressos das mesmas letras. $\mathrm{O}$ número baixo de tentativas de ensino realizadas nesse estudo pode ser resultado do uso do emparelhamento multimodelo, de estímulos compostos e de estímulos familiares, nomeáveis e potencialmente reforçadores.

Em relação ao emparelhamento multimodelo, o estudo de Gomes e de Souza (2008) indicou desempenhos melhores de pessoas com autismo nesse tipo de tarefa, se comparado ao emparelhamento com o modelo típico, sugerindo que este recurso poderia favorecer a aprendizagem de relações entre estímulos visuais por pessoas com autismo. Os dados do estudo atual parecem confirmar e complementar os resultados de Gomes e de Souza (2008), indicando que a aprendizagem das relações arbitrárias entre figuras e palavras impressas foi possível a partir do uso do MTS adaptado com dois ou três modelos simultâneos.

Sobre o uso de estímulos compostos nas tentativas de emparelhamento multimodelo, a literatura especializada indica com muita consistência que pessoas com autismo tendem a manter o foco da atenção em um dos itens do estímulo composto e, consequentemente, podem não considerá-lo com um todo, demonstrando controle restrito de estímulos (Lovaas \& Schreibman, 1971). Apesar disso, dados positivos sobre o uso de estímulos compostos para o ensino de relações arbitrárias entre estímulos visuais foram relatados por Maguire, Stromer, Mackay e Demis (1994), em estudo no qual dois adultos com autismo aprenderam relações entre figuras e apresentaram relações simétricas sem a demonstração de controle restrito de estímulos. Os dados do presente estudo parecem concordar com os resultados de Maguire e colaboradores, ao indicar que os estímulos compostos possibilitaram a aprendizagem das relações arbitrárias entre as figuras $\mathrm{e}$ as palavras impressas (B-C). As dificuldades iniciais apresentadas pelos participantes P2 e P3 podem refletir alguma forma de controle restrito, possivelmente corrigido pelos procedimentos de ensino integrado. Nesses procedimentos, alterou-se a ordem de apresentação das etapas B-D e BC-C, iniciando pela nomeação das figuras, e se estruturou a tentativa com maior número de etapas. Essas novas etapas incluíram a realização da escolha das palavras impressas na presença apenas das figuras (Figura 2, Painel 3) e a conferência dos emparelhamentos realizados (Painel 5). Essas modificações podem ter ampliado o controle de estímulos para aqueles participantes que erravam sistematicamente com o procedimento não integrado e modelos compostos durante a escolha.

Um fator adicional que pode ter favorecido a aprendizagem dos participantes durante as atividades de ensino foi a utilização de itens nomeáveis e reforçadores, sugeridos por cuidadores e variados ao longo do estudo. Esse procedimento pode ter aumentado o engajamento e os acertos dos participantes nas tentativas, semelhante ao descrito em outros estudos de equivalência que utilizaram estímulos nomeáveis e familiares com essa população (LeBlanc, Miguel, Cumming, Goldsmith, \& Carr, 2003; O’Connor et. al, 2009).

Considerando que, nas atividades de ensino, os modelos eram compostos pela figura e pela palavra impressa correspondente, um desempenho preciso nesta etapa não permite afirmar quais relações foram estabelecidas: relações entre figuras e palavras impressas ou relações de identidade entre palavras impressas. Os desempenhos nos testes B-C e C-B, realizados com multimodelos constituídos por elementos unitários (apresentando apenas os elementos figura ou apenas os elementos palavras), são resultados importantes para avaliar o que foi aprendido com as atividades de ensino. Pode-se afirmar que P1 e P2 aprenderam as relações B-C 
(figura-palavra impressa) após a apresentação conjunta dos estímulos compostos (ver Figura 3). Em tese, a relação C-B (palavra impressa - figura) também poderia ser decorrente de ensino direto, conforme afirmação de Stromer et al. (1993) de que a apresentação simultânea dos itens no estímulo composto fornece as condições para o ensino direto da relação entre eles, assim como de sua respectiva relação simétrica (Rebello et al., 2010; Schenk, 1993, 1995). Porém, os resultados de P2 e de P3 indicaram que a aprendizagem consistente da relação B-C não foi acompanhada necessariamente da demonstração da relação C-B em todos os conjuntos de estímulos (ver Figura 3). A ausência de correspondência na aprendizagem dessas relações sugere que a apresentação dos itens no estímulo modelo composto pode ter estabelecido funções fixas e diferentes para os estímulos do conjunto B (possivelmente como modelo) e para os estímulos do conjunto $\mathrm{C}$ (possivelmente como comparação). Assim, a apresentação da tentativa C-B (simétrica) representaria a mudança na função dos estímulos (modelos e comparações), podendo indicar um desempenho que não foi diretamente ensinado e que poderia ou não emergir. Esses resultados sugerem a necessidade de outras investigações a respeito desta hipótese para explicar a inconsistência nos resultados dos Testes B-C e C-B, inclusive com outras populações.

$\mathrm{O}$ desempenho dos participantes nos testes A-C (palavra ditada-palavra impressa) indicou variabilidade e escores mais baixos. Esse resultado pode ser decorrente de dificuldades na aprendizagem de relações auditivo-visuais apresentadas por pessoas com autismo, relatadas pela literatura (Peeters, 1998). Pode também ser decorrente de limitação do procedimento utilizado, que não incluiu o ensino direto de relações auditivo-visuais. Relações A-B (palavra ditada e figura) foram avaliadas antes de iniciar o estudo, mas não foram apresentadas em nenhum outro momento. A nomeação de figuras gera eventos sonoros quando a criança oraliza nessa tarefa e poderiam ter função de estímulos auditivos, porém provenientes de uma resposta do participante e não do experimentador (função instrucional). Os baixos escores no Teste A-C em pelo menos três dos conjuntos de estímulos dos três participantes do presente estudo sugerem a necessidade de se investigar variáveis de procedimento que possam favorecer a emergência dessas relações em indivíduos com autismo. Uma das alternativas poderia ser o uso de consequências específicas (Dube, McIlvane, Maguire, Mackay, \& Stoddard, 1989) compostas que incluíssem um componente auditivo (e.g., Varella \& de Souza, 2014, 2015); por exemplo, ao escolher corretamente uma palavra impressa no ensino BC-C, além das demais consequências programadas, o experimentador poderia dizer a palavra correspondente à figura e a palavra impressa (cf. Pilgrim, 2011; Varella \& de Souza, 2014, 2015).

Em relação à nomeação de palavras impressas $(\mathrm{C}$ D), todos os participantes mostraram consistentemente a emergência dessas discriminações (ou operantes verbais cada palavra falada sob controle de um estímulo impresso diferente) não ensinadas diretamente. $\mathrm{O}$ delineamento de linha de base múltipla, que monitorava a nomeação das palavras ao longo do estudo, permitiu observar que os participantes não eram capazes de nomear as palavras impressas no início das unidades e passaram a nomeá-las somente após o ensino de cada conjunto de estímulos. O delineamento experimental mostrou efeito inequívoco do ensino sobre a nomeação oral diante das palavras impressas. Na maioria dos casos, ocorreu a manutenção da nomeação das palavras em testes subsequentes. O termo nomeação está sendo preferido ao termo comportamento textual (Skinner, 1957) porque, sob as condições do estudo, não há como identificar se o comportamento ocorria sob controle ponto-a-ponto dos elementos textuais, ou se as palavras estavam sendo tratadas apenas figurativamente.

Os resultados do estudo indicam que o ensino da relação BC-C em tentativas de emparelhamento multimodelo e o ensino da relação B-D favoreceram a aprendizagem da relação B-C e a aprendizagem possivelmente indireta das relações $\mathrm{C}-\mathrm{B}$, A-C (desempenhos mais baixos) e C-D. Considerando que os participantes eram capazes de relacionar palavras ditadas e figuras (A-B), demonstrado em pré-testes iniciais, a emergência da relação A-C, decorrente de A-B e B-C, apresenta a propriedade de transitividade, enquanto a emergência de C-B, decorrente de B-C, indica a simetria. Desta forma, os dados deste estudo sugerem desempenhos emergentes e formação de classes de estímulos equivalentes (Sidman, 1971; Sidman \& Tailby, 1982) para P2 (Conjunto 3 das Unidades 1 e 2) e para P3 (Conjunto 3 da Unidade 1 e Conjunto 1 da Unidade 2). Os testes realizados neste estudo foram, entretanto, mais frágeis do que os recomendados para afirmações conclusivas sobre a formação de classes. Testes B-C e C-B foram realizados com o procedimento de MTS adaptado e não foram avaliadas, no decorrer do estudo, as relações A-B.

Pode-se concluir que os procedimentos utilizados no estudo foram efetivos para promover habilidades rudimentares de leitura, especialmente de leitura oral de palavras impressas (C-D), o que é bastante relevante, especialmente para aprendizes que estão no começo do processo de alfabetização e que apresentam dificuldades em aprender pelos métodos de ensino convencionais, como é o caso de pessoas com autismo (Gomes \& de Souza, 2013; Peeters, 1998). Contudo, somente a aprendizagem dessas habilidades rudimentares não é suficiente para formar leitores plenamente eficientes; para isso é necessário o desenvolvimento de metodologias que possibilitem a leitura recombinativa generalizada com compreensão de palavras (de Rose et al., 1996; Hanna et al., 2011; Mueller, Olmi, \& Saunders, 2000), o que deve ser objeto de outras investigações.

\section{REFERENCIAS}

American Psychiatric Association. (2013). Diagnostic and statistical manual of mental disorders (Fifth ed.). Arlington, VA: American Psychiatric Publishing.

Carr, D., Wilkinson, K. M., Blackman, D., \& McIlvane, W. J. (2000). Equivalence classes in individuals with minimal verbal repertoires. Journal of the Experimental Analysis of Behavior, 74 (1), 101-114. 
Carter, D. E., \& Eckerman, D. A. (1975). Symbolic matching by pigeons: Rate of learning complex discriminations predicted from simple discriminations. Science, 187, 662-664.

Cumming, W. W., \& Berryman, R. (1961). Some data on matching behavior in the pigeon. Journal of the Experimental Analysis of Behavior, 4, 281-284.

Cumming, W. W., \& Berryman, R. (1965). The complex discriminated operant: Studies of matching-to-sample. Em D. I. Mostofsky (Org.), Stimulus generalization (pp. 284-330). Stanford, CA.: Stanford University Press.

de Rose, J. C. (2004). Emparelhamento com modelo e suas aplicações. In C. N. de Abreu, \& H. J. Guilhardi (Eds.), Terapia comportamental e cognitivocomportamental: Práticas clínicas (pp. 215-225). São Paulo: Roca.

de Rose, J. C., de Souza, D. G., \& Hanna, E. S. (1996). Teaching reading and spelling: Exclusion and stimulus equivalence. Journal of Applied Behavior Analysis, 27, 451-469.

Duarte, G. M., \& de Rose, J. C. (2006). A aprendizagem simbólica em crianças com déficit atencional. Revista Brasileira de Educação Especial, 12, 331-350.

Dube, W. V. (1996). Teaching discrimination skills to persons with mental retardation. In C. Goyos, M. A. Almeida, \& D. G. de Souza (Eds.), Temas em Educação Especial III (pp. (pp. 73-96). São Carlos: EDUFSCar.

Dube, W., \& McIlvane, W. (1995). Stimulus-reinforcer relations and emergent matching-to- sample. The Psychological Record, 45, 591-612.

Dube, W. V., McIlvane, W. J., Maguire, R. W., Mackay, H. A., \& Stoddard, L. T. (1989). Stimulus class formation and stimulus-reinforcer relations. Journal of the Experimental Analysis of Behavior, 51(1), 65-76.

Eikeseth, S., \& Smith, T. (1992). The development of function and equivalence classes in high-functioning autistic children: The role of naming. Journal of the Experimental Analysis of Behavior, 58, 123-133.

Ghezzi, P., Williams, W., \& Carr, J. (1999). Autism: Behavior analytic perspectives. Reno: Context Press.

Gomes, C. G., \& de Souza, D. G. (2008). Desempenho de pessoas com autismo em tarefas de emparelhamento com o modelo por identidade: Efeitos da organização dos estímulos. Psicologia: Reflexão e Crítica, 21(3), 412-423.

Gomes, C. G., \& de Souza, D. G. (2013). Transtornos do espectro do autismo de alto funcionamento e aprendizagem de conteúdos acadêmicos. In: W. Camargos Jr. (Org.). Síndrome de Asperger e outros transtornos do espectro do autismo de alto funcionamento: Da avaliação ao tratamento (pp. 301314). Belo Horizonte: ArteSã.

Hanna, E. S., Kohlsdorf, M., Quinteiro, R. S., de Melo, R. M., de Souza, D. G., de Rose, J. C., \& McIlvane, W. J. (2011). Recombinative reading derived from pseudoword instruction in a miniature linguistic system. Journal of the Experimental Analysis of Behavior, 95, 21-40.
Kazdin, A. E. (1982). Single-case research designs: Methods for clinical and applied settings. New York: Oxford University Press.

Kelly S., Green G., \& Sidman M. (1998). Visual identity matching and auditory visual matching: A procedural note. Journal of Applied Behavior Analysis, 31(2), 237-243.

LeBlanc, L., Miguel, C. F., Cumming, A., Goldsmith, T., \& Carr, J. E. (2003). The effects of three stimulusequivalence testing conditions on emergent U. S. geography relations of children diagnosed with autism. Behavioral Interventions, 18, 279-289.

Leon, V. C., Bosa, C. A., Hugo, C. N., \& Hutz, C. (2004). Propriedades psicométricas do Perfil Psicoeducacional Revisado: PEP-R. Avaliação Psicológica, 3(1), 39-52.

Lovaas, O. I., \& Schreibman, L. (1971). Stimulus overselectivity of autistic children in a two-stimulus situation. Behaviour Research and Therapy, 9, 305310 .

Maguire, R. W., Stromer, R., Mackay, H. A., \& Demis, C. A. (1994). Matching to complex samples and stimulus class formation in adults with autism and young children. Journal of Autism and Developmental Disorders, 24, 753-772.

Martin, G., \& Pear, J. (1978) Behavior modification: What it is and how to do it. Englewood Cliffs: Prentice-Hall.

Mueller, M. M., Olmi, D. J., \& Saunders, K. J. (2000). Recombinative generalization of within-syllable units in prereading children. Journal of Applied Behavior Analysis, 33, 515-531.

Nation, K., Clarke, P., Wright, B., \& Williams, C. (2006). Patterns of reading ability in children with autism spectrum disorder. Journal of Autism and Developmental Disorders, 36, 911-919.

O'Connor, J., Rafferty, A., Barnes-Holmes, D., \& BarnesHolmes, Y. (2009). The role of verbal behavior, stimulus nameability and familiarity on the equivalence performances of autistic and normally developing children. The Psychological Record, 59 (10), 53-74.

Peeters, T. (1998). Autismo: Entendimento teórico e intervenção educacional. Rio de Janeiro: Cultura Médica.

Pereira, A., Riesgo, R. S., \& Wagner, M. B. (2008). Autismo infantil: Tradução e validação da Childhood Autism Rating Scale para uso no Brasil. Jornal de Pediatria, 84(6),487-494.

Pilgrim, C. (2011, November). Stimulus equivalence via simple discrimination training: A translational approach. In: E. Arntzen (Chair). Invited presentation at the ABAI Sixth International Conference, Granada, Spain.

Rebello, M., Yu, L. S., Bertoldo, M., Duckur, R., Vernucio, R., \& Debert, P. (2010). Ensino de ideogramas com base no procedimento matching de identidade com estímulos compostos. Revista Brasileira de Análise do Comportamento, 6(2), 203201.

Saunders, R., \& Green, G. (1999). Discrimination analysis of training-structure effects on stimulus equivalence 
outcomes. Journal of the Experimental Analysis of Behavior, 72, 117-137.

Schenk, J. J. (1993). Emergent conditional discrimination in children: Matching to compound stimuli. Quarterly Journal of Experimental Psychology, 46(4), 345-365.

Schenk, J. J. (1995). Complex stimuli in nonreinforced simple discrimination task: Emergent simple and conditional discriminations. The Psychological Record, 45, 477-494.

Schopler, E., Reichler, R. J., Bashford, A., Lansing, M. D., \& Marcus, L. M. (1990). Individualized assessment and treatment for autistic and developmentally disabled children: Psychoeducational profile-revised (PEP-R). Austin, TX: PRO-ED.

Schopler, E., Reichler, J. R., \& Renner, C. (1988). CARSThe Childhood Autism Rating Scale. Los Angeles: Westerm Pychological Services.

Serna, R. W., Dube, W. V., \& McIlvane, W. J. (1997). Assessing same/different judgments in individuals with severe intellectual disabilities: A status report. Research in Developmental Disabilities, 18, 343-368.

Sidman, M. (1971). Reading and auditory-visual equivalences. Journal of Speech and Hearing Research, 14, 5-13.

Sidman, M. (1985). Aprendizagem-sem-erros e sua importância para o ensino do deficiente mental. Psicologia, 11 (3), 1-15.

Sidman, M. (1994). Equivalence relations and behavior: A research story. Boston: Authors Cooperative.

Sidman, M., \& Tailby, W. (1982). Conditional discrimination vs. matching to sample: An expansion of the testing paradigm. Journal of the Experimental Analysis of Behavior, 37, 5-22.

Skinner, B. F. (1957). Verbal behavior. Engelwood Cliffs, NJ: Prentice Hall.

Stromer, R., McIlvane, W. J., Dube, W. V., \& Mackay, H. A. (1993). Assessing control by elements of complex stimuli in delayed matching to sample. Journal of the Experimental Analysis of Behavior, 59, 83-102.

Stromer, R., McIlvane, W., \& Serna R. (1993). Complex stimulus control and equivalence. The Psychological Record, 43, 585-598.

TEACCH. (1992). Independent tasks: Work activities for students with autism and other visual learners. Division of Department of Psychiatry School of Medicine, University of North Carolina, Chapel Hill.

Varella, A. A. B., \& de Souza, D. G. (2014). Emergence of auditory-visual relations from a visual-visual baseline with auditory-specific consequences in individuals with autism. Journal of the Experimental Analysis of Behavior, 102, 139-149.

Varella, A. A. B., \& de Souza, D. G. (2015). Using classspecific compound consequences to teach dictated and printed letter relations to a child with autism. Journal of Applied Behavior Analysis, 48, 1-5.

Vause, T., Martin, G. L., Yu, C. T., Marion, C., \& Sakko, G. (2005). Teaching equivalence relations to individuals with minimal verbal repertoires: Are visual and auditory-visual discriminations predictive of stimulus equivalence? The Psychological Record, 55, 197-218.

Wilkinson, K. M., Rosenquist, C., \& McIlvane, W. J. (2009). Exclusion learning and emergent symbolic category formation in individuals with severe language impairments and intellectual disabilities. The Psychological Record, 59, 187-206.

Williams, G., Pérez-González, L. A., \& Queiroz, A. (2005). Using a combined blocking procedure to teach color discrimination to a child with autism. Journal of Applied Behavior Analysis, 38, 555-558.

Recebido em 16/12/2014 Aceite final em 10/06/2015

Editor Associado: Edson Massayuki Huziwara 